\title{
Proteomic and Phenotypic Analyses of a Putative Glycerol-3-Phosphate Dehydrogenase Required for Virulence in Acidovorax citrulli
}

\author{
Minyoung Kim ${ }^{1}$, Jongchan Lee ${ }^{1}$, Lynn Heo ${ }^{1}$, Sang Jun Lee (iD) ${ }^{2 *}$, and Sang-Wook Han (iD ${ }^{1 *}$ \\ ${ }^{1}$ Department of Plant Science and Technology, Chung-Ang University, Anseong 17546, Korea \\ ${ }^{2}$ Department of Systems Biotechnology, Chung-Ang University, Anseong 17546, Korea
}

(Received on December 9, 2020; Revised on December 28, 2020; Accepted on December 29, 2020)

Acidovorax citrulli $(A c)$ is the causal agent of bacterial fruit blotch (BFB) in watermelon, a disease that poses a serious threat to watermelon production. Because of the lack of resistant cultivars against $B F B$, virulence factors or mechanisms need to be elucidated to control the disease. Glycerol-3-phosphate dehydrogenase is the enzyme involved in glycerol production from glucose during glycolysis. In this study, we report the functions of a putative glycerol-3-phosphate dehydrogenase in $A c$ (GlpdAc) using comparative proteomic analysis and phenotypic observation. A glpdAc knockout mutant,

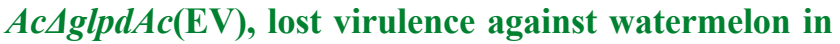
two pathogenicity tests. The putative 3D structure and amino acid sequence of GlpdAc showed high similarity with glycerol-3-phosphate dehydrogenases from other bacteria. Comparative proteomic analysis revealed that many proteins related to various metabolic pathways, including carbohydrate metabolism, were affected by

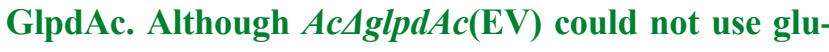
cose as a sole carbon source, it showed growth in the

*Co-corresponding authors.

S. J. Lee

Phone) +82-31-670-3356, FAX) +82-2-675-3108

E-mail) sangjlee@cau.ac.kr

S.-W. Han

Phone)+82-31-670-3150, FAX) +82-2-670-8845

E-mail)swhan@cau.ac.kr

ORCID

Sang Jun Lee

https://orcid.org/0000-0002-2803-753X

Sang-Wook Han

https://orcid.org/0000-0002-0893-1438

(c) This is an Open Access article distributed under the terms of the Creative Commons Attribution Non-Commercial License (http:// creativecommons.org/licenses/by-nc/4.0) which permits unrestricted noncommercial use, distribution, and reproduction in any medium, provided the original work is properly cited.

Articles can be freely viewed online at www.ppjonline.org. presence of glycerol, indicating that GlpdAc is involved in glycolysis. $A c \Delta g l p d A c(E V)$ also displayed higher cellto-cell aggregation than the wild-type bacteria, and tolerance to osmotic stress and ciprofloxacin was reduced and enhanced in the mutant, respectively. These results indicate that GlpdAc is involved in glycerol metabolism and other mechanisms, including virulence, demonstrating that the protein has pleiotropic effects. Our study expands the understanding of the functions of proteins associated with virulence in $A c$.

Keywords : bacterial fruit blotch, glycerol-3-phosphate dehydrogenase, virulence, watermelon

Handling Editor : Chang-Jin Park

Acidovorax citrulli (Ac), previously known as Pseudomonas pseudoalcaligenes subsp. citrulli, causes bacterial fruit blotch (BFB) disease in cucurbit crops such as watermelon (Latin and Rane, 1990; Sowell and Schaad, 1979). The disease was first observed in 1989 in Florida, USA, and has since spread throughout the US and other countries, including Korea (Burdman and Walcott, 2012). Under favorable conditions, the crop losses caused by BFB have been greater than $90 \%$ on marketable crops. The disease generally begins with contaminated seeds because $A c$ is known as a seed-borne pathogen (Latin and Hopkins, 1995). The initial symptoms are water-soaked lesions on cotyledons and wilt development in seedlings. In fruit, the infected surfaces also show water-soaked lesions, which expand, leading to necrosis of the infected fruit (Latin and Hopkins, 1995). Thus, BFB is a major threat to the production of watermelon. Despite its negative impact on the industry, resistant cultivars of watermelon have not been developed. Therefore, virulence factors or mechanisms in $A c$ have to be elucidated to control the disease. 
There was a focus on controlling BFB spread in the 1990s, and various studies regarding virulence factors and mechanisms in $A c$ were subsequently carried out. It was elucidated that twitching motility and biofilm formation, which are mediated by type IV pili, are indispensable for $A c$ virulence (Bahar et al., 2009). It was also reported that quorum sensing and ferric uptake systems are involved in the regulation of its virulence (Johnson and Walcott, 2013; Liu et al., 2019). Additionally, research focusing on the type III secretion system is actively ongoing (JiménezGuerrero et al., 2019). Recently, Kim et al. (2020) reported that CmpAc, which is a bifunctional chorismate mutase and prephenate dehydratase, is involved in virulence and other mechanisms in Ac. However, other genes and mechanisms that are related to virulence in $A c$ have not been definitively identified.

Glycerol-3-phosphate (G3P) dehydrogenase is a cytosolic enzyme involved in carbohydrate and lipid metabolism, and it controls the conversion process between dihydroxyacetone phosphate and G3P (Guindalini et al., 2010). This G3P dehydrogenase has been shown to be the key enzyme that produces glycerol from glucose during glycolysis (Hao et al., 2015). Further, G3P dehydrogenase plays an important role in the virulence of Pseudomonas aeruginosa (Daniels et al., 2014). In Magnaporthe oryzae, the deletion of G3P dehydrogenase had negative effects on fungal development and virulence (Shi et al., 2018). The function of G3P dehydrogenase in Colletotrichum gloeosporioides is also related to conidiation (Wei et al., 2004). Thus, G3P and glycerol assimilation and carbon utilization through G3P dehydrogenase play important roles in pathogenicity.

In this study, we report the functions of a putative G3P dehydrogenase in $A c$ (GlpdAc) in virulence and other biological mechanisms using a G3P dehydrogenase knockout mutant $(A c \triangle g l p d A c)$. To predict and characterize the functions of GlpdAc, the label-free shotgun proteomics following clusters of orthologous groups (COGs) was carried out. In addition to comparative proteomic analysis, diverse phenotypic assays were also conducted to elucidate the roles of GlpaAc in $A c$.

\section{Materials and Methods}

Bacterial strains and growth conditions. For this study, the $A c$ group II strain, KACC17005, whose genome information has been fully sequenced (Park et al., 2017), was used as the wild-type strain. $A c$ strains were grown in TSB (tryptic soy broth, $30 \mathrm{~g} / \mathrm{l}$ ) with appropriate antibiotics at $28^{\circ} \mathrm{C}$. For generating the plasmid constructs, Escherichia coli strain DH5 $\alpha$ was used, and for cloning Tn5-inserted DNA fragments, the E. coli strain EC100D was used (Epicentre, Madison, WI, USA). E. coli strains were grown in LB (Luria-Bertani; $1 \%$ tryptone, $0.5 \%$ yeast extract, and $1 \% \mathrm{NaCl}$ ) with appropriate antibiotics. The final antibiotic concentrations in the media were as follows: kanamycin, $50 \mu \mathrm{g} / \mathrm{ml}$; rifampicin, $50 \mu \mathrm{g} / \mathrm{ml}$; gentamicin, $10 \mu \mathrm{g} / \mathrm{ml}$; and ampicillin, $100 \mu \mathrm{g} / \mathrm{ml}$.

Generation of $A c \Delta g l p d A c$ and its complementary strain. To generate the knockout of $g l p d A c$, the EZ-Tn $5<$ R6K $\gamma$ ori/ KAN-2> Insertion Kit (Lucigen, Middleton, WI, USA) was used. Following the kit procedure, Tn5 was randomly inserted on one site of the $A c$ genome, and this insertion was verified using Tn5-specific primers 5 '-GGATCGCGATGGATATGTTCT-3' and 5'-AACAGGAATCGAATGCAACC-3'. Genomic DNA was extracted, and the Tn5-disrupted DNA fragment was cloned following the manufacturer's protocol (Lucigen). After cloning the DNA fragment, its DNA sequence was verified by Sanger sequencing with the following primers: forward (KAN-2F), 5'-ACCTACAACAAAGCTCTCATCAACC-3' and reverse (R6KAN-2R), 5'-CTACCCTGTTGGAACACCTACATCT-3' (Lucigen). The confirmed strain was named $A c \Delta g l p d A c$. To create the construct for the complemented strain, the open reading frame of $g l p d A c$ was amplified using the following gene-specific primers: 5'-CTCGAGATGAAGATCATCGT-3' and 5'-AAGCTTCAGTGGTGGTGGTGGTGGTGGCAGGAGCGCAA-3'. The amplicon was cloned into the pGem-T Easy vector (Promega, Madison, WI, USA), generating pGem-GlpdAc, and the cloned DNA was confirmed by Sanger sequencing. The construct was digested with restriction enzymes $X h o \mathrm{I}$ and HindIII, and the digested DNA fragment was subcloned into the pBBR1-MCS5 vector, which is a broad host range vector containing the lac $Z$ promoter (Kovach et al., 1995), generating pBBR1-GlpdAc. The generated plasmid was introduced into $A c \triangle g l p d A c$ and selected on tryptic soy agar (TSA) with appropriate antibiotics. The selected transformant was confirmed using PCR with GlpdAc-specific primers and called $A c \Delta g l p d A c(\mathrm{GlpdAc})$. To control for vector side-effects, an empty pBBR1-MCS5 vector was also introduced into $A c$ and $A c \Delta g l p d A c$, generating $A c(\mathrm{EV})$ and $A c \Delta g l p d A c(\mathrm{EV})$ strains, respectively. The transformants were selected on TSA with appropriate antibiotics and then confirmed by PCR with the following pBBR1MCS5-specific primers: 5'-CAGGGTTTTCCCAGTCACGA-3' and 5'-ATGCTTCCGGCTCGTATGTT-3'. The generated plasmids and strains are described in Supple- 
mentary Table 1.

Prediction of 3D structure. To predict the putative 3D structure of GlpdAc, the deduced amino acid sequence of GlpdAc was evaluated through the I-TASSER server (Roy et al., 2010), and the PDB file was obtained. This file was imported into the PyMOL program (Molecular Graphics System, San Carlos, CA, USA), generating the putative 3D structure. For comparison, the 3D structure of GpsA, a G3P dehydrogenase in Coxiella burnetii, was also generated using the same method.

Pathogenicity assay. Watermelon (Citrullus lanatus var. vulgaris) line SBA, which was kindly provided by Partner Seed Company (Gimje, Korea), was used for virulence testing. A germinated-seed inoculation was used according to a previously established method with slight modifications (Bahar et al., 2009). For effective inoculation, a pre-germination step was added. To prepare the germinated seeds for inoculation, they were placed on sterilized filter papers with $4 \mathrm{ml}$ sterilized water and incubated at $28^{\circ} \mathrm{C}$ for 2 days; germination was confirmed by visual observation. The $A c$ strains were grown on TSA with antibiotics at $28^{\circ} \mathrm{C}$. The bacteria were collected and suspended in $10 \mathrm{mM} \mathrm{MgCl}_{2}$ to an optical density at $600 \mathrm{~nm}\left(\mathrm{OD}_{600}\right)$ of 0.3 (approximately $10^{8}$ colony forming units $[\mathrm{cfu}] / \mathrm{ml}$ ) and then diluted to $10^{6}$ $\mathrm{cfu} / \mathrm{ml}$ with $10 \mathrm{mM} \mathrm{MgCl}{ }_{2}$. Ten pre-germinated seeds were incubated in $20 \mathrm{ml}$ of bacterial suspension and gently agitated for $1 \mathrm{~h}$. After incubation, the inoculated seeds were sown in a 50-pot tray with sterilized soil and sealed with a tray cover for 2 days. The planted seeds were then grown in a growth chamber and checked for disease severity for 7 days. Disease severity was evaluated using a $0-2$ scale $(0$, no symptoms; 1 , water-soaked region; 2 , seedling wilt) as described previously (Kim et al., 2020; Song et al., 2020). A disease index was then calculated as follows:

Disease index $=[$ (Numbers of normal plants $) \times 0+($ Numbers of plants with water-soaked regions $) \times 1+($ Number of wilted plants $) \times 2] /($ Total number of plants $)$

The infiltration of watermelon leaves was performed according to a previous protocol (Bahar et al., 2011). The pre-germinated watermelon seeds were sown and grown for 2 weeks, at which time the seedlings had acquired four true leaves. The $A c$ strains were suspended in $10 \mathrm{mM}$ $\mathrm{MgCl}_{2}$ to an $\mathrm{OD}_{600}$ of 0.3 and diluted to $10^{5} \mathrm{cfu} / \mathrm{ml}$ with 10 $\mathrm{mM} \mathrm{MgCl} 2$. The undersides of first and second leaves were inoculated with bacterial suspension using a $3 \mathrm{ml}$ needleless syringe. For counting CFUs, the inoculated leaves were punched using cork-borers $(0.4 \mathrm{~cm}$ diameter $)$, and two punches were ground in $200 \mu \mathrm{l}$ sterilized water using a tissue grinder. The leaf extracts were serially diluted (10fold). The diluted suspensions were spotted onto TSA with antibiotics and incubated at $28^{\circ} \mathrm{C}$ for 2 days. Cfus were determined 0, 2, 4 6, and 8 days after inoculation. Data from three biological replicates were collected.

Proteomics analysis. The proteomics protocols and conditions, including protein extraction, peptide generation, liquid chromatography-tandem mass spectrometry (LCMS/MS), peptide quantification/identification, comparison of identified proteins, and COG classification, were as reported previously (Park et al., 2020). Briefly, three biological replicates of the $A c$ strains (a total of six samples) were grown in TSB to an $\mathrm{OD}_{600}$ of 0.5 and collected by centrifugation. Total soluble proteins were then extracted from the bacteria, and peptides were generated. A split-free nano-LC system (EASY-nLC II, Thermo Fisher Scientific, Waltham, MA, USA) combined with an LTQ Velos Pro instrument (Thermo Fisher Scientific) was used for the proteomics analysis. Mass spectra from the LC-MS/MS analysis were identified using the $A c$ strain KACC17005 database from the National Center for Biotechnology Information, and the identified peptides were quantified using Thermo Proteome Discoverer 1.3 (ver. 1.3.0.399) and the SEQUEST program. After peptide quantification, protein levels in $A c$ and $A c \Delta g l p d A c$ were compared. Finally, differentially expressed proteins were categorized by COG analysis. Student's t-tests $(P<0.05)$ were used for statistical comparisons.

Bacterial growth in rich and minimal media. For bacterial growth assay in TSB medium, $A c(\mathrm{EV}), A c \Delta g l p d A c(\mathrm{EV})$, and $A c \Delta g l p d A c(\mathrm{GlpdAc})$ were harvested, washed twice with sterilized water, and suspended in TSB to an $\mathrm{OD}_{600}$ of $0.3\left(10^{8} \mathrm{cfu} / \mathrm{ml}\right)$. The bacterial suspensions were then diluted to $10^{5} \mathrm{cfu} / \mathrm{ml}$ with TSB and measured for $96 \mathrm{~h}$ at intervals of $12 \mathrm{~h}$ using a spectrophotometer. To determine the effect of carbon sources on the $A c$ strains, bacterial growth was assayed in M9 minimal medium containing glucose or glycerol (Hames et al., 2009). For the assay, M9 minimal medium ( $5 \times \mathrm{M} 9$ salts, $200 \mathrm{ml} ; 1 \mathrm{M} \mathrm{MgSO}_{4}, 2 \mathrm{ml}$; $1 \mathrm{M} \mathrm{CaCl}_{2}, 0.1 \mathrm{ml}$ in 1 1) with $20 \mathrm{~mL}$ of $20 \%$ glycerol or $20 \%$ glucose was used. The bacteria were grown on TSA, harvested, washed twice, and suspended in $10 \mathrm{mM} \mathrm{MgCl}{ }_{2}$ to an $\mathrm{OD}_{600}$ of 0.05 . The bacterial growth was measured for 10 days at $24 \mathrm{~h}$ intervals using a spectrophotometer. Three biological replicates were used in this assay.

Cell-cell aggregation assay. The assay for cell-cell ag- 
gregation was carried out as previously described (Bahar et al., 2009). $A c$ strains were grown in TSA, washed, and suspended in TSB. The bacterial suspension was adjusted to an $\mathrm{OD}_{600}$ of 0.1 (4 ml in $15 \mathrm{ml}$ tube), incubated for $12 \mathrm{~h}$ at $28^{\circ} \mathrm{C}$, and kept without shaking at $22^{\circ} \mathrm{C}$ for $2 \mathrm{~h}$. After the bacterial cells had settled at the bottom of the tube, the absorbance at $600 \mathrm{~nm}\left(\mathrm{OD}_{\mathrm{s}}\right)$ of the upper $1 \mathrm{ml}$ of liquid was measured with a spectrophotometer. The residual bacteria were completely dispersed by vortexing and the absorbance at $600 \mathrm{~nm}$ was measured $\left(\mathrm{OD}_{\mathrm{t}}\right)$. The cell-cell aggregation rate was calculated as $\left[\left(\mathrm{OD}_{\mathrm{t}}-\mathrm{OD}_{\mathrm{s}}\right) \times 100\right] / \mathrm{OD}_{\mathrm{t}}$. For this assay, six biological replicates were collected.
Osmotic and antibiotic stress tolerance assays. To estimate bacterial tolerance to stress conditions, treatments with $2.5 \% \mathrm{NaCl}$ for osmotic stress, and $0.1 \mu \mathrm{g} / \mathrm{ml}$ ciprofloxacin for antibiotic stress were used. For osmotic stress, the bacterial strains were grown, harvested, and adjusted to an $\mathrm{OD}_{600}$ of 0.3 . The bacteria were then treated with $2.5 \%$ $\mathrm{NaCl}$ for $10 \mathrm{~min}$. For antibiotic stress, the bacteria strains were harvested, suspended at an $\mathrm{OD}_{600}$ of 0.1 , and exposed to $0.1 \mu \mathrm{g} / \mathrm{ml}$ ciprofloxacin for $2 \mathrm{~h}$. The treated and untreated bacterial suspensions were serially diluted and plated on TSA with appropriate antibiotics. After incubation for 2 days at $28^{\circ} \mathrm{C}$, the numbers of colonies were counted. The ratio of stress-treated bacterial cell count to untreated
A

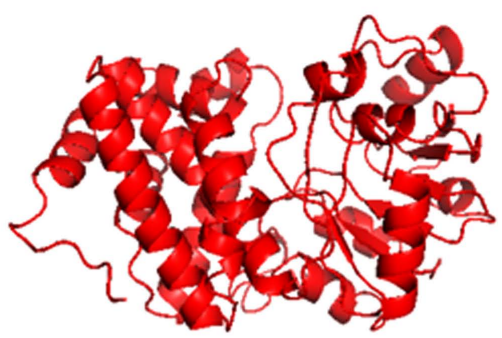

B

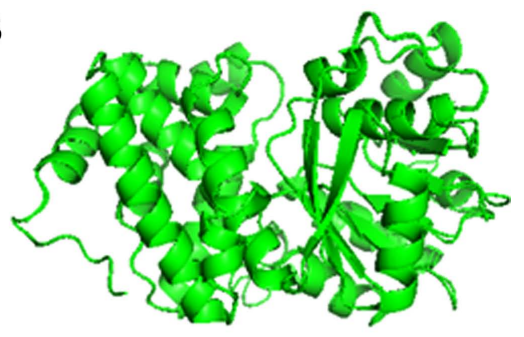

C

ATG93866 ABM34399 TQK64598 0.JV61281

ATG93866 ABM34399 TQK64598 0JV61281

ATG93866 ABM34399 TQK64598 0.JV61281

ATG93866 ABM34399 TQK64598 0JV61281

ATG93866 ABM34399 TQK64598 0JV61281

ATG93866 ABM34399 TQK64598 0JV61281
MKI I VFGAGAWGTAMALSAAAHPAGHAVTLWARDGRQADAMQAARQNARYLPGI AFPAAL MKI I VFGAGAWGTAMALSAAAHPAGHAVTLWARDGRQADAMQAARQNARYLPGI AFPAAL MKI I VLGAGAWGSALAMSAAEHPAGHAVTLWARDAGQAEAMRAARQNARYLPGI GFPPSL MKI I VLGAGAWGSAVAMSAAQHPAGHAVTLWGRDAAQAQSMRAQAQNARYLPGI ALPPSL $* * * * *: * * * * * *: *: *: * * * * * * * * * * * * *, * *, * *:: *: * * * * * * * * * *,: *: *$

ALASGAPSGALASCRADLA IVATPMSGLRGMLEELRDATI PVAWLCKGFEAVPAGGETAA ALASGAPSGALASCRADLA IVATPMSGLRGMLEELRDAT I PVAWLCKGFEAVPAGGETAA TVADGPFGALL--PGADLVIVATPMAALRGMLRALTGCTAPVAVLCKGFEAVPPGADSAS SVVDGDFRALL--PGADLVIVATPMAALRGMLLALRGCTAPVAWLCKGFEAVPAGANSAS $* * *, * * * * * *: . * * * * * * \ldots * * * * * * * * * * * * * * *,:: *:$

QGLMAHE I CSQVAPRLRAGALSGPSFALEAAQGRPTALVAASRDAHVRELLVEAFHGPTL QGLMAHE ICSQVAPRLRAGALSGPSFALEAAQGRPTALVAASRDAHVRELLVEAFHGPTL FGLLAHEVQAQVAPDLVAGVFSGPSFAQEVAQGQPTALVAASAHAAVRDALVKAFHSPSV FGLLAHEVQAQVAPYL I AGVFSGPSFAQEVALGQPTALVAASPHAQVRDALVSAFHSPSV $* *: * * *:: * * * * * * *,: * * * * * * *, * *: * * * * * * * *, * * *: * *, * * *, *:$ :

RVYANED I VGVEVGGAVKNVLA I ATGLCDGLDLGTNARAAL I TRGLAEMSRLGLALGARA RVYANED I VGVEVGGAVKNVLAI ATGLCDGLDLGTNARAAL I TRGLAEMSRLGLALGARA RVYANED I VGVEVGGAVKNVLAI ATGLCDGLSLGLNARAAL I TRGLAEMTRLGLALGARP RVYANED I VGVEVGGAVKNVLAI ATGLCDGLALGLNARAAL I TRGLAEMTRLGLALGARP $* * * * * * * * * * * * * * * * * * * * * * * * * * * * * * * * * * * * * * * * * * * * * * * ; * * * * * * * * * *$

ETFMGLSGLGDLVLTATGDLSRNRRVGLALARGLTLDQAVESLGHVAEGVYSARTVVRRA ETFMGLSGLGDLVLTATGDLSRNRRVGLALARGLTLDQAVESLGHVAEGVYSARTVYRRA DTFMGLSGLGDLVLTATGDLSRNRRVGMLLAQGHTLAQAVESLGHVAEGVYSARTVVQRA DTFMGLSGLGDLVLTATGDLSRNRRVGMLLAQGQTLAQALESLGHVAEGVYSARTVVQRA $: * * * * * * * * * * * * * * * * * * * * * * * * * *$ : $* *: * * * * *: * * * * * * * * * * * * * * * * *: * *$

GQLGVDMP I AREVVALLDGRSTASDAVARLMGRSPAAELRSC GQLGVDMP I AREVVALLDGRSTASDAVARLMGRSPAAELRSC RLLGVDMP I AEAAVALLDGRLQPAEALAQLMGRGPRVEA--QTLGVDMP I AEAAVALLDGRLQPAEAVAQLMGRCPRGEL--$* * * * * * * * * * * * * * * * * \quad \cdots * * * * * * * * * * * *$

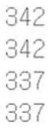

Fig. 1. Predicted 3D structures and amino acid sequence alignments for GlpdAc. Predicted 3D structures of GlpdAc (A) and glycerol-3-phosphate dehydrogenase (GpsA) (B) from Coxiella burnetii were generated using the I-TASSER sever and PyMol program. (C) Deduced amino acid sequence alignments of GlpdAc (ATG93866), NAD(P)H-dependent glycerol3-phosphate dehydrogenase from Ac strain AAC00-1 (ABM34399), glycerol-3-phosphate dehydrogenase $(\mathrm{NAD}(\mathrm{P})+)$ from Nocardioides $\mathrm{sp}$. SLBN-35 (TGK64598), and glycerol3-phosphate dehydrogenase from Burkholderiales bacterium 64-34 (OJV61281). These results were obtained using the Clustal Omega Program. The '*', ':', and '.' indicate identical residues, conserved substitutions, and semi-conserved substitutions, respectively. 
bacterial cell count was calculated for each strain and stress treatment. For this study, at least three biological replicates were used for each strain.

\section{Results}

Predicted 3D-protein structure and sequence comparison. By screening a Tn5 insertion library, we identified one mutant with reduced virulence in watermelon and confirmed that one gene (accession no. ATG93866), identified as G3P dehydrogenase, was disrupted by Tn5. The predicted 3D structure of ATG93866 is similar to that of G3P dehydrogenase (GpsA) from C. burnetii, which is the causal agent of $\mathrm{Q}$ fever in humans (Fig. 1A and B). As shown in Fig. 1C, the deduced amino acid sequence of ATG93866 has high similarity with the NAD(P)H-dependent GlpdAc strain AAC00-1, G3P dehydrogenase in Nocardioides sp.
SLBN-35, and G3P dehydrogenase in Burkholderiales bacterium 64-34. Therefore, ATG93866 was named GlpdAc (glycerol-3-phosphate dehydrogenase in $A c$ ).

The requirement of GlpdAc for virulence in $A c$. To investigate the role of GlpdAc in virulence, germinatedseed inoculation and leaf infiltration were performed. $A c(\mathrm{EV})$ (wild-type strain carrying an empty vector), $A c \Delta g l p d A c(\mathrm{EV})$ ( $g l p d A c$ knockout mutant carrying an emphajeoty vector), and the complemented strain, $A c \Delta g l p d A c(\mathrm{GlpdAc})(A c \Delta g l p d A c$ carrying $g l p d A c$ on $\mathrm{pB}-$ BR1MCS5) were tested. In the germinated-seed inoculation, the disease index of $A c(\mathrm{EV})$ continuously increased and reached a value of 2 at 7 days after inoculation (DAI). In contrast, $A c \Delta g l p d A c(\mathrm{EV})$ did not cause disease, with a disease index was 0 at 7 DAI (Fig. 2A), demonstrating that GlpdAc is indispensable for virulence in $A c$. The virulence
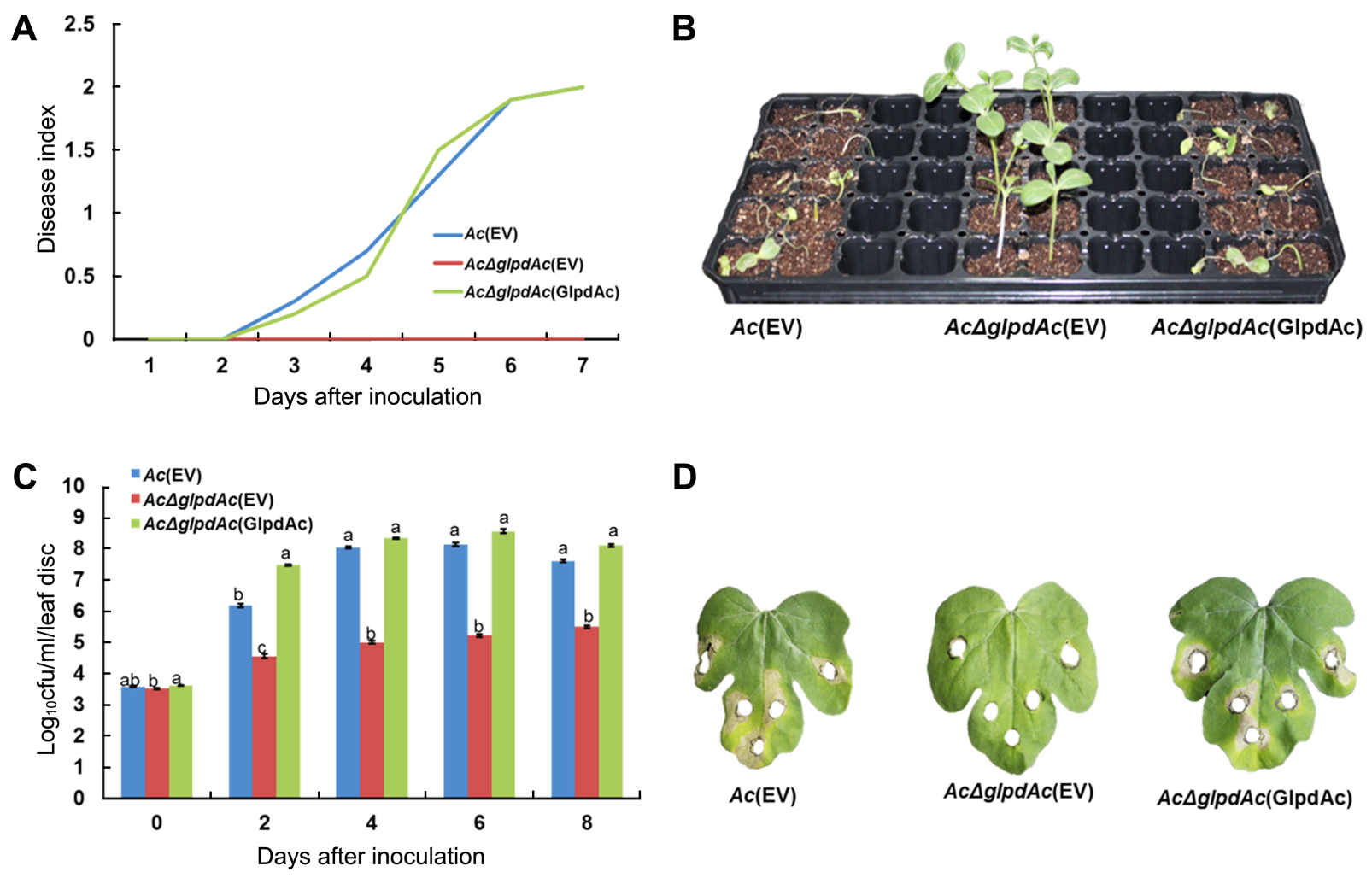

D
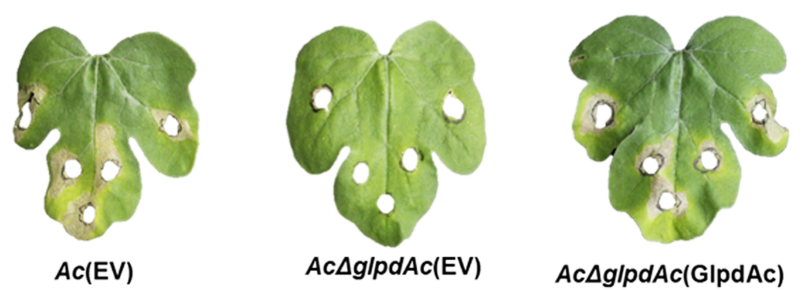

$A c \Delta g / p d A c(\mathrm{GIpdAc})$

Fig. 2. Pathogenicity test for $A c(\mathrm{EV}), A c \Delta g l p d A c(\mathrm{EV})$, and $A c \Delta g l p d A c(\mathrm{GlpdAc})$ on watermelon. (A) Disease index from the seedgerminated method. Disease index $=[$ (Number of normal plants $) \times 0+($ Number of plants with water-soaked regions $) \times 1+($ Number of wilted plants $) \times 2] /($ Total number of plants). (B) Symptoms of infected seedlings in the seed-germinated method. The photograph was taken 7 days after inoculation (DAI). The index scale of watermelon infected by $A c(\mathrm{EV})$ and $A c \Delta g l p d A c(\mathrm{GlpdAc})$ was 2 and this of $A c \Delta g l p d A c(\mathrm{GlpdAc})$ was 0 at 7 DAI. (C) Bacterial multiplication in watermelon after leaf infiltration. Two-week-old watermelon plants at the 4-true-leaf stage were inoculated in their first and second leaves via needleless syringe. In the inoculated area, leaf discs were punched. Through serial dilution, $\log _{10}$ cfus were measured. Cfu counting was conducted for 8 days, in 2-day intervals. The different letters indicated significant differences $(P<0.05)$ using ANOVA with Tukey's HSD post-hoc analysis. (D) Images from the leaf infiltration. The punched areas indicate where the bacterial suspension infiltrated. The photographs were taken 8 days after inoculation. 
A
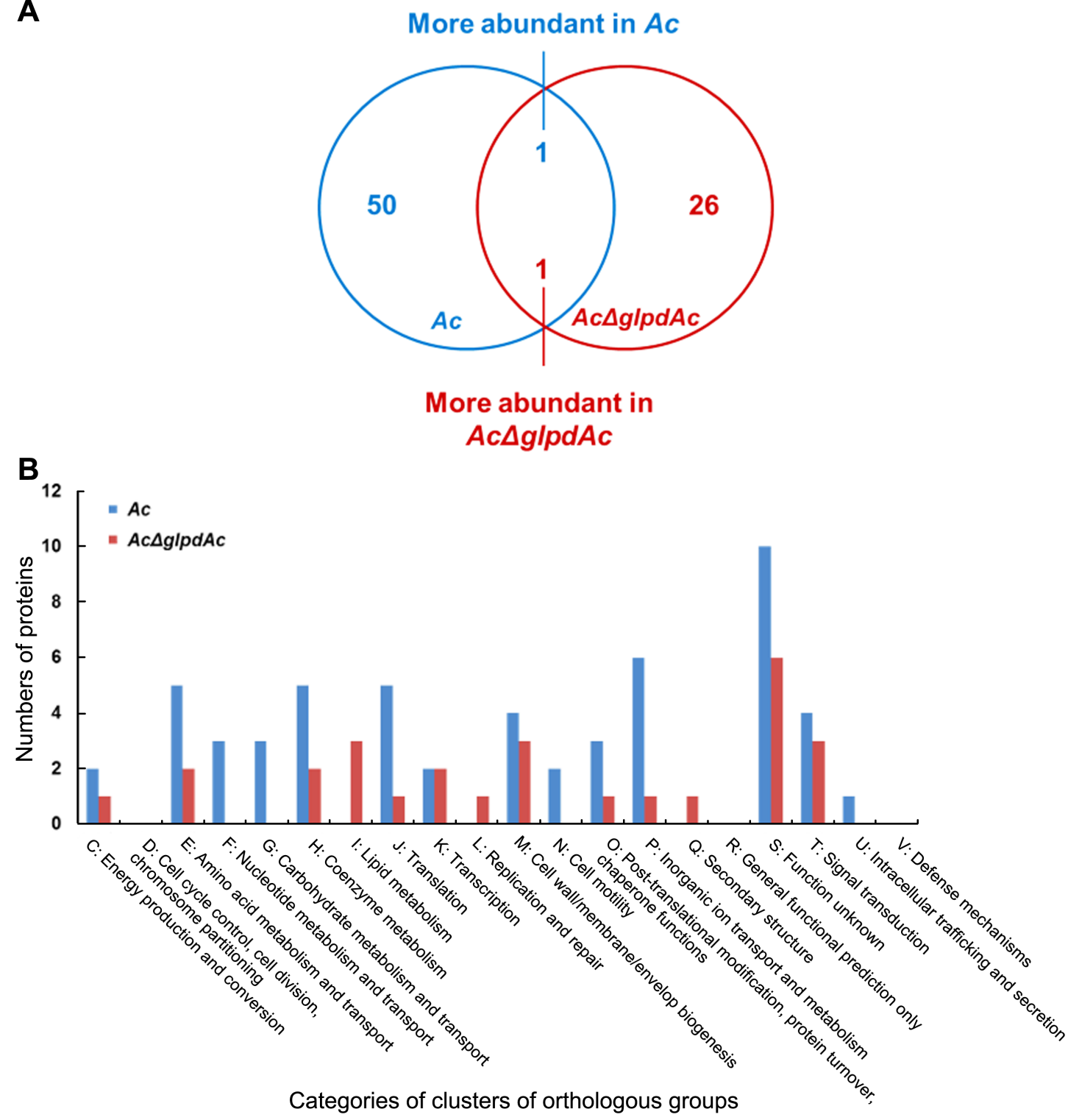

Fig. 3. Comparison of proteome analysis of Acidovorax citrulli (Ac) and AcAglpdAc. (A) Venn diagram reveals that abundance of 51 and 27 proteins were changed (>2-fold) in comparative proteomic analysis. Fifty and 26 proteins were uniquely detected in $A c$ and $A c \Delta g l p d A c$, respectively. One protein was more abundant in $A c$ and in $A c \Delta g l p d A c$. (B) Classification of clusters of orthologous groups of differentially abundant proteins ( $>2$-fold) by proteomics analysis.

of $A c \Delta g l p d A c(\mathrm{GlpdAc})$ was similar to that of $A c(\mathrm{EV})$, indicating that there were no positional effects by the insertion of Tn5 in GlpdAc. As shown in Fig. 2B, watermelon infected by $A c \Delta g l p d A c(\mathrm{EV})$ did not show disease symptoms. The results of the leaf-infiltration experiments were similar to those of the seed-germinated inoculation. Although the $A c \Delta g l p d A c(\mathrm{EV})$ population slightly increased during the observation period, it was significantly lower than that of $A c(\mathrm{EV})$ (Fig. 2C). However, the growth of the complemented strain was not different from that of $A c(\mathrm{EV})$. Simi- lar to the seed-germinated inoculation, $\operatorname{Ac} \Delta \operatorname{glp} d A c(\mathrm{EV})$ did not induce symptoms on the leaves of watermelon (Fig. 2D). However, leaves infiltrated with $A c(\mathrm{EV})$ and $A c \Delta g l p d A c(\mathrm{GlpdAc})$ showed necrosis near the site of inoculation.

Comparative proteomics analysis in $A c$ and $A c \Delta g l p d A c$. It is clear that GlpdAc is involved in virulence. In addition, it has been shown that one protein related to biochemical pathways can produce pleiotropic effects in bacteria, in- 
cluding the alteration of virulence and other physiological mechanisms (Felgner et al., 2016; Kim et al., 2020). Therefore, we carried out comparative proteomics to postulate biological and cellular mechanisms related to GlpdAc using the wild-type and $A c \Delta g l p d A c$ strains. In three biological replicates of the $A c$ strain, 751, 757, and 752 proteins were found from 59,405, 58,831, and 59,569 peptide spectral matches (PSMs), respectively; 726, 695, and 722 proteins from 59,367, 58960, and 59,261 PSMs, respectively, were identified from the Ac $\Delta g l p d A c$ strain (Supplementary Table 2). Among them, 696 and 658 proteins were commonly detected in the three biological replicates of $A c$ and $A c \Delta g l p d A c$, respectively, and these proteins were used for the comparative analysis. It was found that 51 and 27 proteins were more abundant ( $>2$-fold) in $A c$ and $A c \Delta g l p d A c$, respectively (Fig. 3A). Seventy-eight proteins were subjected to COG classification. The COG analysis revealed that proteins classified in groups $\mathrm{F}$ (nucleotide metabolism and transport), $\mathrm{G}$ (carbohydrate metabolism and transport), $\mathrm{N}$ (cell motility), and $\mathrm{U}$ (intracellular trafficking and secretion) were uniquely found in the $A c$ strain, and proteins categorized in groups I (lipid metabolism), L (replication and repair), and Q (secondary structure) were detected only in Ac $\triangle g l p d A c$ (Fig. 3B, Supplementary Tables 3 and 4). Proteins belonging to other categories were mostly abundant in the $A c$ strain. Interestingly, fructose 1/6-bisphosphatase, C4-dicarboxylate $\mathrm{ABC}$ transporter substrate-binding protein, twitching motility protein PilT, and universal stress protein were only detected in $A c$. These results indicate that GlpdAc is involved in diverse cellular processes in $A c$, including carbohydrate metabolism and cell wall/membrane biogenesis.

Involvement of GlpdAc with glycolysis in $A c$. Sequence comparison and proteomics analysis of GlpdAc reveal that the protein may be associated with glucose/glycerol metabolism. Therefore, bacterial growth in the presence of glucose or glycerol as a sole carbon source was investigated. First, we examined the growth of $A c(\mathrm{EV}), A c \Delta g l p d A c(\mathrm{EV})$, and $A c \Delta g l p d A c(\mathrm{GlpdAc})$ in a rich medium to test the effect of GlpdAc on bacterial multiplication (Fig. 4A). During the measurement period, the $\mathrm{OD}_{600}$ values of the three strains were not significantly different, indicating that GlpdAc is not associated with bacterial reproduction. As shown in Fig. 4B, the growth of $A c \Delta g l p d A c(\mathrm{EV})$ barely increased in M9 medium with glucose, but the complemented strain showed similar growth to that of $A c(\mathrm{EV})$. Notably, the growth of $A c \Delta g l p d A c(\mathrm{EV})$ in $\mathrm{M} 9$ medium supplemented with glycerol was not different from that of $A c(\mathrm{EV})$ and $A c \Delta g l p d A c(\mathrm{GlpdAc})$ (Fig. 4C). These data suggest that the
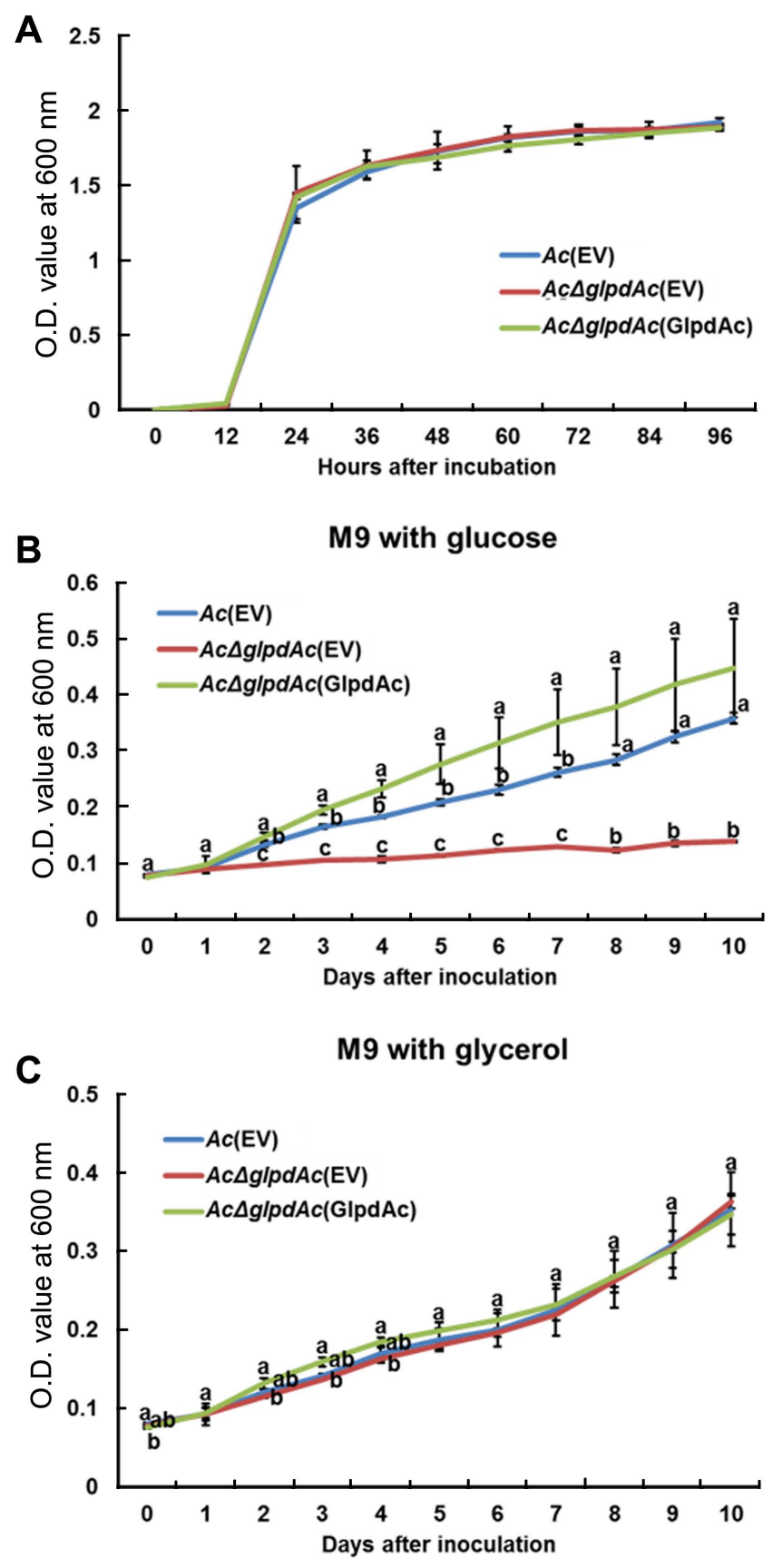

Fig. 4. Growth of Acidovorax citrulli (Ac) strains in rich medium and M9 supplemented with glucose or glycerol. (A) Growth of three strains, $A c(\mathrm{EV}), A c \Delta g l p d A c(\mathrm{EV})$, and $A c \Delta g l p d A c(\mathrm{GlpdAc})$, in tryptic soy broth medium was measured for 4 days at 12-h intervals using a spectrophotometer $\left(\mathrm{OD}_{600}\right)$. There was no statistical difference among strains at each time point. Growth of the three strains was examined in M9 medium supplemented with glucose (B) or glycerol (C) for 10 days at 24-h intervals using a spectrophotometer $\left(\mathrm{OD}_{600}\right)$. Three biological replicates were analyzed. The different letters indicate significantly different values $(P<0.05)$ using ANOVA with Tukey's HSD post-hoc analysis. Error bars indicate standard deviations. 


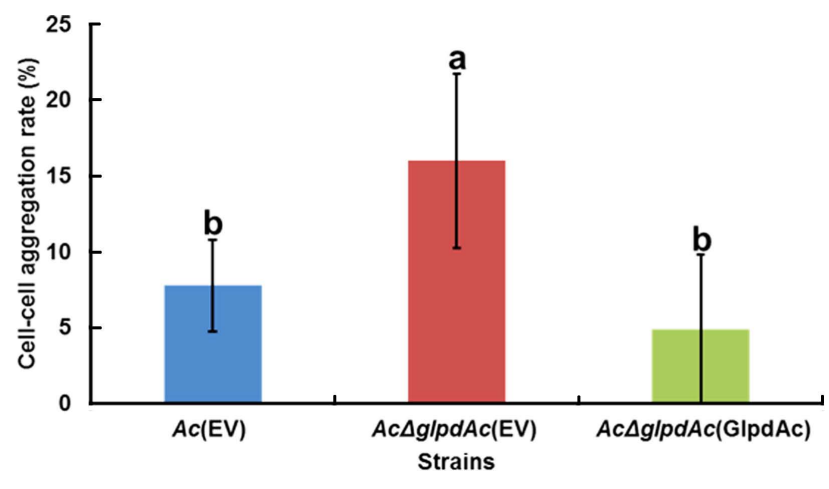

Fig. 5. Cell-cell aggregation assay. The bacterial suspension was adjusted to an $\mathrm{OD}_{600}$ of 0.1 and incubated without mixing at $22^{\circ} \mathrm{C}$ for $2 \mathrm{~h}$. The supernatant was carefully removed and its turbidity measured by a spectrophotometer (ODs). The remaining pellet was completely resuspended by vortexing and its turbidity was measured by a spectrophotometer (ODt). The cell-cell aggregation percentage was calculated as: $\left[\left(\mathrm{OD}_{\mathrm{t}}-\mathrm{OD}_{\mathrm{s}}\right) \times 100\right] / \mathrm{OD}_{\mathrm{t}}$. The different letters represent significant differences using ANOVA $(P$ $<0.05)$ with Tukey's HSD post-hoc test. Error bars indicate standard deviations. Six biological replicates were used for this assay.

function of GlpdAc is related to glycolysis.

Enhanced cell-cell aggregation in $A c \Delta g l p d A c(E V)$. In the comparative proteomics analysis, the abundance of diverse proteins related to cell wall/membrane/envelope, including PilT, which is associated with cell aggregation (Bahar et al., 2009), was altered (Supplementary Tables 3 and 4). To examine the effect of GlpdAc on cell aggregation, a bacterial cell sedimentation assay was conducted using fully grown $A c(\mathrm{EV}), A c \Delta g l p d A c(\mathrm{EV})$, and $A c \Delta g l p d A c(\mathrm{GlpdAc})$.

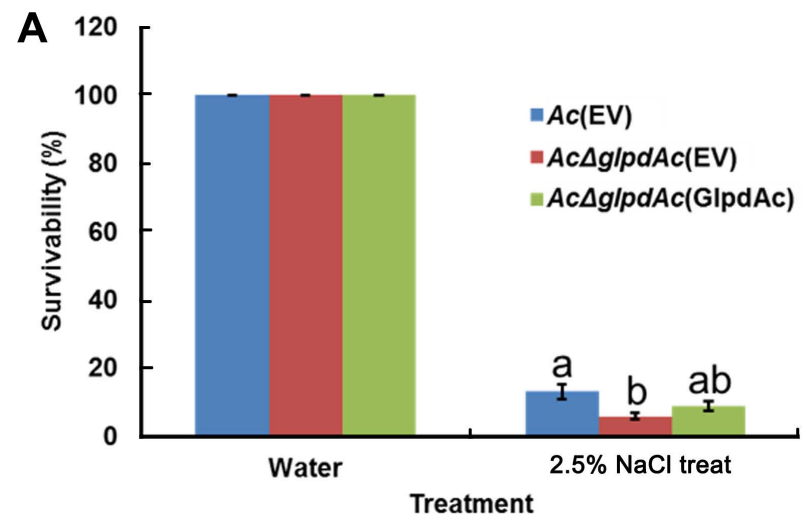

As shown in Fig. 5, the bacteria that remained in suspension after allowing the medium to stand for $2 \mathrm{~h}$ and those that had pelleted were quantified, and the cell-cell aggregation ratio was calculated. $A c(\mathrm{EV})$ showed a cell-cell aggregation ratio of $7.76 \%$. The ratio for $A c \Delta g l p d A c(\mathrm{EV})$ was $16 \%$, which was approximately 2 -fold higher than that for $A c(\mathrm{EV})$. The cell-cell aggregation in $A c \Delta g l p d A c(\mathrm{GlpdAc})$ was restored to the level in $A c(\mathrm{EV})$.

Altered tolerance to antibiotic and osmotic stress in $A c \Delta g l p d A c(E V)$. In the proteomics analysis, a universal stress protein (ATG96448) was found to be more abundant in $A c$ than in $A c \Delta g l p d A c$. Proteins associated with cell membrane/wall/envelope biogenesis were also abundantly detected in the comparative proteomics analysis. Thus, the tolerance to osmotic stress and antibiotics in $A c(\mathrm{EV}), A c \Delta g l p d A c(\mathrm{EV})$, and $A c \Delta G g l p d A c(\mathrm{GlpdAc})$ was investigated. After treatment with $2.5 \% \mathrm{NaCl}, 6.61 \%$ of $A c \Delta g l p d A c(\mathrm{EV})$ had survived, compared to $16.7 \%$ of $A c(\mathrm{EV})$ (Fig. 6A), indicating that the mutant was less tolerant to osmotic stress. Survival was restored to wild-type levels in the complemented strain. Notably, with $0.1 \mu \mathrm{g} / \mathrm{ml}$ ciprofloxacin treatment, $A c \Delta g l p d A c(\mathrm{EV})$ displayed higher survival (7.79\%) than both $A c(\mathrm{EV})(5.93 \%)$ and Ac $\Delta g l p d A c($ GlpdAc) (4.22\%) (Fig. 6B).

\section{Discussion}

In many pathogens, glucose and glycerol metabolism has been implicated in virulence. For example, Mycoplasma glycerol metabolism is essential to its cell cytotoxicity

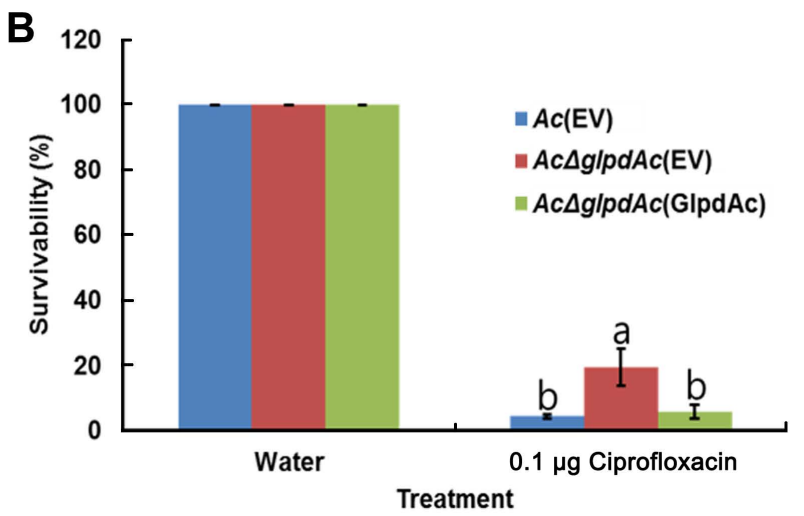

Fig. 6. Tolerance test against osmotic stress and ciprofloxacin. Tolerance against two stress conditions, $2.5 \% \mathrm{NaCl}$ for $10 \mathrm{~min}$ (A) and 0.1 $\mu \mathrm{g} / \mathrm{ml}$ ciprofloxacin for $2 \mathrm{~h}$ (B), was investigated. Treated bacterial suspensions were serially diluted and plated on tryptic soy agar containing appropriate antibiotics. Water was used as a control. Survivability was established from the numbers of bacteria after treatment to those in the water control using the colony counting method. Different letters indicate significantly different means as determined by ANOVA $(P<0.05)$, followed by Tukey's HSD post-hoc analysis. Error bars indicate standard errors. Three biological replicates were used for each strain. 
and is involved in its virulence (Blötz and Stülke, 2017; Schmidl et al., 2011). This glycerol metabolism is mediated by G3P dehydrogenase, which is known as a key enzyme for glycerol production from glucose during glycolysis (André et al., 1991). In P. aeruginosa, the analogous G3P dehydrogenase plays a role in producing virulence factors (Daniels et al., 2014). In addition, in the rice blast fungus, $M$. oryzae, conidia formation and disease development were affected by G3P dehydrogenase, and the G3P dehydrogenase knockout mutant was less virulent than the wild-type strain (Shi et al., 2018). Thus, the G3P dehydrogenase that is indispensable for glycerol production from glucose is an important component of virulence. In agreement with these previous studies, $A c \Delta g l p d A c(\mathrm{EV})$ characterized in our study also showed significantly reduced disease symptom development and bacterial populations on watermelon, indicating that GlpdAc contributes to virulence in $A c$. Nevertheless, $A c \Delta g l p d A c(\mathrm{EV})$ showed little difference compared to $A c(\mathrm{EV})$ and $A c \Delta g l p d A c(\mathrm{GlpdAc})$ in bacterial growth assays in TSB. These results suggest that the reduction in virulence of $A c \Delta g l p d A c(\mathrm{EV})$ is not related to bacterial multiplication.

Comparative proteomics indicated that GlpdAc is associated with diverse metabolic processes, including a carbohydrate metabolic pathway. Especially, fructose 1/6-bisphosphatase (ATG94560), which is a glycolytic enzyme (Spoering et al., 2006), was detected only in Ac. Furthermore, proteins categorized in groups $\mathrm{E}$ (amino acid metabolism and transport) and $\mathrm{H}$ (coenzyme metabolism) were found to be abundant in $A c$. In addition, proteins involved in other metabolic processes were more abundant in $A c$ than in $A c \Delta g l p d A c$. Thus, GlpdAc is likely associated with carbohydrate metabolism as well as other metabolic processes. In the growth assay, $A c \Delta g l p d A c(\mathrm{EV})$ did not grow in the presence of glucose but did grow in the presence of glycerol. These data indicate that GlpdAc is a key enzyme involved in the glucose-dependent pathway of carbohydrate metabolism, rather than the glycerol-dependent pathway. The sequence analysis of GlpdAc also supported this observation. Because GlpdAc encodes a putative G3P dehydrogenase, GlpdAc likely catalyzes the conversion of glucose to glycerol during glycolysis. In addition, the twitching motility protein PilT, which belongs to group $\mathrm{N}$ (cell motility), was detected in $A c$ but not in $A c \Delta g l p d A c$. In a previous study, the group I $A c$ strain knockout mutant of PilT showed high cell-cell aggregation (Bahar et al., 2009). Similarly, $A c \Delta g l p d A c(\mathrm{EV})$ displayed higher cell-cell aggregation than $A c(\mathrm{EV})$ or $A c \Delta g l p d A c(\mathrm{GlpdAc})$ in our study. PilT is involved in the retraction of type IV pili, and the loss of this function could negatively affect virulence.
Thus, our proteomics analysis and phenotypic observations reveal that impaired mechanisms caused by the loss of GlpdAc function trigger abnormal phenotypes, which may contribute to $A c$ virulence.

In E. coli, G3P dehydrogenase is a monotopic enzyme that is bound to the inner membrane and is involved in energy regulation (Yeh et al., 2008). According to a previous study, G3P dehydrogenase also participates in sustainable cell formation in E. coli (Spoering et al., 2006). Therefore, it can be predicted that the altered mechanisms in $A c \Delta g l p d A c(\mathrm{EV})$ could reduce tolerance to external stress. For example, the deletion mutant of G3P dehydrogenase in Saccharomyces cerevisiae showed enhanced sensitivity to osmotic stress conditions (Albertyn et al., 1994). Similar to this observation, $A c \Delta g l p d A c(\mathrm{EV})$ was less tolerant to growth in $2.5 \% \mathrm{NaCl}$ than both $A c(\mathrm{EV})$ and $A c \Delta g l p d A c(\mathrm{GlpdAc})$. In contrast, the impaired function of GlpdAc enhanced tolerance to ciprofloxacin. It was also reported that the loss of G3P dehydrogenase function in $P$. aeruginosa increased cell formation persistence, resulting in enhanced tolerance to antibiotic stress (Shuman et al., 2018). Our proteomics analysis also supports these phenotypic observations because the abundance of many proteins involved in cell membrane or wall processes was changed. These results suggest that impaired GlpdAc alters metabolic processes and the expression of various proteins, resulting in varying persistence to different stressors. In both plants and humans, glycerol-3-phosphate dehydrogenase (GAPDH) is reported as a target of plant defense-related hormone salicylic acid (SA) (Choi et al., 2015; Tian et al., 2015). In humans, SA's interaction with GAPDH suppressed its nuclear translocation and subsequent cell death. On the other hands, SA inhibited Tomato bushy stunt virus (TBSV), a single-stranded RNA virus, replication in tobacco plants by inhibiting the binding of cytosolic GAPDH to the negative (-)RNA strand of TBSV. Our study suggests that GlpdAc, which encodes GAPDH, is an important virulence factor in $A c$. Thus it will be very interesting whether GlpdAc can be a target of SA, and SA's binding to GlpdAc can reduce virulence in $A c$.

Taken together, the functions of GlpdAc were characterized using sequence analysis, comparative proteomics, and diverse phenotypic assays in this study. Consequently, GlpdAc was shown to be involved in pathogenicity, glucose metabolism, cell-cell aggregation, and tolerance to osmotic stress and ciprofloxacin antibiotics in Ac. But, specific molecular mechanisms of GlpdAc regarding various phenotypes are still elusive, and it has to be elucidated in further study. The lack of availability of resistant cultivars against BFB highlights the need to characterize virulence 
mechanisms and factors in $A c$ to control the disease. Our study findings indicate that GlpdAc is a potential target to develop antivirulence agents for suppressing the virulence of $A c$.

\section{Conflicts of Interest}

No potential conflict of interest relevant to this article was reported.

\section{Acknowledgments}

This work was supported by the National Research Foundation of Korea (NRF) grant funded by the Korean government (MSIT) (No. NRF-2020R1A2C1013040) (to S.W. Han).

\section{Electronic Supplementary Material}

Supplementary materials are available at The Plant Pathology Journal website (http://www.ppjonline.org/).

\section{References}

Albertyn, J., Hohmann, S., Thevelein, J. M. and Prior, B. A. 1994. GPD1, which encodes glycerol-3-phosphate dehydrogenase, is essential for growth under osmotic stress in Saccharomyces cerevisiae, and its expression is regulated by the high-osmolarity glycerol response pathway. Mol. Cell. Biol. 14:41354144.

André, L., Hemming, A. and Adler, L. 1991. Osmoregulation in Saccharomyces cerevisiae: studies on the osmotic induction of glycerol production and glycerol-3-phosphate dehydrogenase (NAD ${ }^{+}$). FEBS Lett. 286:13-17.

Bahar, O., Goffer, T. and Burdman, S. 2009. Type IV pili are required for virulence, twitching motility, and biofilm formation of Acidovorax avenae subsp. citrulli. Mol. Plant-Microbe Interact. 22:909-920.

Bahar, O., Levi, N. and Burdman, S. 2011. The cucurbit pathogenic bacterium Acidovorax citrulli requires a polar flagellum for full virulence before and after host-tissue penetration. Mol. Plant-Microbe Interact. 24:1040-1050.

Blötz, C. and Stülke, J. 2017. Glycerol metabolism and its implication in virulence in Mycoplasma. FEMS Microbiol. Rev. 41:640-652.

Burdman, S. and Walcott, R. 2012. Acidovorax citrulli: generating basic and applied knowledge to tackle a global threat to the cucurbit industry. Mol. Plant Pathol. 13:805-815.

Choi, H. W., Tian, M., Manohar, M., Harraz, M. M., Park, S.W., Schroeder, F. C., Snyder, S. H. and Klessig, D. F. 2015. Human GAPDH is a target of aspirin's primary metabolite salicylic acid and its derivatives. PLoS ONE 10:e0143447.
Daniels, J. B., Scoffield, J., Woolnough, J. L. and Silo-Suh, L. 2014. Impact of glycerol-3-phosphate dehydrogenase on virulence factor production by Pseudomonas aeruginosa. Can. J. Microbiol. 60:857-863.

Felgner, S., Frahm, M., Kocijancic, D., Rohde, M., Eckweiler, D., Bielecka, A., Bueno, E., Cava, F., Abraham, W.-R., Curtiss, R. 3rd, Häussler, S., Erhardt, M. and Weiss, S. 2016. aroAdeficient Salmonella enterica serovar Typhimurium is more than a metabolically attenuated mutant. mBio 7:e01220-16.

Guindalini, C., Lee, K. S., Andersen, M. L., Santos-Silva, R., Bittencourt, L. R. A. and Tufik, S. 2010. The influence of obstructive sleep apnea on the expression of glycerol-3-phosphate dehydrogenase I gene. Exp. Biol. Med. 235:52-56.

Hames, C., Halbedel, S., Hoppert, M., Frey, J. and Stülke, J. 2009. Glycerol metabolism is important for cytotoxicity of Mycoplasma pneumoniae. J. Bacteriol. 191:747-753.

Hao, G., Chen, H., Gu, Z., Zhang, H., Chen, W. and Chen, Y. Q. 2015. Metabolic engineering of Mortierella alpina for arachidonic acid production with glycerol as carbon source. Microb. Cell Fact. 14:205.

Jiménez-Guerrero, I., Pérez-Montaño, F., Da Silva, G. M., Wagner, N., Shkedy, D., Zhao, M., Pizarro, L., Bar, M., Walcott, R., Sessa, G., Pupko, T. and Burdman, S. 2019. Show me your secret(ed) weapons: a multifaceted approach reveals a wide arsenal of type III-secreted effectors in the cucurbit pathogenic bacterium Acidovorax citrulli and novel effectors in the Acidovorax genus. Mol. Plant Pathol. 21:17-37

Johnson, K. L. and Walcott, R. R. 2013. Quorum sensing contributes to seed-to-seedling transmission of Acidovorax citrulli on watermelon. J. Phytopathol. 161:562-573.

Kim, M., Lee, J., Heo, L. and Han, S.-W. 2020. Putative bifunctional chorismate mutase/prephenate dehydratase contributes to the virulence of Acidovorax citrulli. Front. Plant Sci. 11:569552.

Kovach, M. E., Elzer, P. H., Hill, D. S., Robertson, G. T., Farris, M. A., Roop, R. M. 2nd. and Peterson, K. M. 1995. Four new derivatives of the broad-host-range cloning vector $\mathrm{pB}$ BR1MCS, carrying different antibiotic-resistance cassettes. Gene 166:175-176.

Latin, R. X. and Hopkins, D. L. 1995. Bacterial fruit blotch on watermelon: the hypothetical exam question becomes reality. Plant Dis. 79:761-765.

Latin, R. X. and Rane, K. K. 1990. Bacterial fruit blotch of watermelon in Indiana. Plant Dis. 74:331.

Liu, J., Tian, Y., Zhao, Y., Zeng, R., Chen, B., Hu, B. and Walcott, R. R. 2019. Ferric uptake regulator (FurA) is required for Acidovorax citrulli virulence on watermelon. Phytopathology 109:1997-2008.

Park, H.-J., Lee, J., Kim, M. and Han, S.-W. 2020. Profiling differentially abundant proteins by overexpression of three putative methyltransferases in Xanthomonas axonopodis pv. glycines. Proteomics 20:e1900125.

Park, H.-J., Seong, H. J., Sul, W. J., Oh, C.-S. and Han, S.-W. 2017. Complete genome sequence of Acidovorax citrulli 
strain KACC17005, a causal agent for bacterial fruit blotch on watermelon. Korean J. Microbiol. 53:340-341.

Roy, A., Kucukural, A. and Zhang, Y. 2010. I-TASSER: a unified platform for automated protein structure and function prediction. Nat. Protoc. 5:725-738.

Schmidl, S. R., Otto, A., Lluch-Senar, M., Piñol, J., Busse, J., Becher, D. and Stülke, J. 2011. A trigger enzyme in Mycoplasma pneumoniae: impact of the glycerophosphodiesterase GlpQ on virulence and gene expression. PLoS Pathog. 7:e1002263.

Shi, Y., Wang, H., Yan, Y., Cao, H., Liu, X., Lin, F. and Lu, J. 2018. Glycerol-3-phosphate shuttle is involved in development and virulence in the rice blast fungus Pyricularia oryzae. Front. Plant Sci. 9:687.

Shuman, J., Giles, T. X., Carroll, L., Tabata, K., Powers, A., Suh, S.-J. and Silo-Suh, L. 2018. Transcriptome analysis of a Pseudomonas aeruginosa sn-glycerol-3-phosphate dehydrogenase mutant reveals a disruption in bioenergetics. Microbiology 164:551-562.

Song, Y.-R., Hwang, I. S. and Oh, C.-S. 2020. Natural variation in virulence of Acidovorax citrulli isolates that cause bacterial fruit blotch in watermelon, depending on infection routes.
Plant Pathol. J. 36:29-42.

Sowell, G. Jr. and Schaad, N. W. 1979. Pseudomonas pseudoalcaligenes subsp. citrulli on watermelon: seed transmission and resistance of plant introductions. Plant Dis. Rep. 63:437441.

Spoering, A. L., Vulić, M. and Lewis, K. 2006. GlpD and PlsB participate in persister cell formation in Escherichia coli. J. Bacteriol. 188:5136-5144.

Tian, M., Sasvari, Z., Gonzalez, P. A., Friso, G., Rowland, E., Liu, X. M., van Wijk, K. J., Nagy, P. D. and Klessig, D. F. 2015. Salicylic acid inhibits the replication of tomato bushy stunt virus by directly targeting a host component in the replication complex. Mol. Plant-Microbe Interact. 28:379-386.

Wei, Y., Shen, W., Dauk, M., Wang, F., Selvaraj, G. and Zou, J. 2004. Targeted gene disruption of glycerol-3-phosphate dehydrogenase in Colletotrichum gloeosporioides reveals evidence that glycerol is a significant transferred nutrient from host plant to fungal pathogen. J. Biol. Chem. 279:429-435.

Yeh, J. I., Chinte, U. and Du, S. 2008. Structure of glycerol3-phosphate dehydrogenase, an essential monotopic membrane enzyme involved in respiration and metabolism. Proc. Natl. Acad. Sci. U. S. A. 105:3280-3285. 